\title{
112 \\ Designing Virtual Environments for Usability
}

\author{
Kulwinder Kaur \\ Centre for HCI Design, City University, Northampton Square \\ London EC1V 0HB \\ UNITED KINGDOM \\ K.Kaur@soi.city.ac.uk
}

ABSTRACT The development of HCI guidelines for virtual environments, is reported, using interaction modelling.

KEYWORDS virtual reality, interaction models, interaction design

\section{INTRODUCTION}

Virtual Environments (VEs) are a novel interface type offering new possibilities for human-computer interaction. Notable features of VEs include their structure as 3D graphical models which the user navigates through and interacts with, typically using natural interaction styles, such as viewpoint control modelled on human head movements.

However, guidance on how to design usable VEs are hard to find, even though significant usability problems have been reported (e.g. Rushton and Wann 1993). The objective of this dissertation research is to develop guidelines for designing VEs from a usability perspective. The hypotheses are:

H1: There is a need for interface design guidance specifically for VEs.

H2: General patterns of interaction with VEs can be predicted.

H3: Design properties required for interaction can be predicted using these general patterns $(\mathrm{H} 2)$.

H4: Interaction can be improved by implementing these design properties $(\mathrm{H} 3)$.

H5: The design properties (H3) can be presented in a usable form to support VE interface design and evaluation.

\section{H1: NEED FOR VE DESIGN SUPPORT}

To test hypothesis 1, two studies were carried out (see Kaur et al., 1996) to investigate the usability of VE interfaces and current design practice for VEs.

In the first study, usability was investigated through an evaluation of the Royal Navy's Virtual Submarine using observation of interaction sessions. Trainee submariners experienced major interaction problems, such as getting and maintaining a suitable viewing angle, navigating through tight areas, losing whereabouts after getting too close to objects (nose against the wall) and recognising interactive hot-spots in the environment (see figure 1).

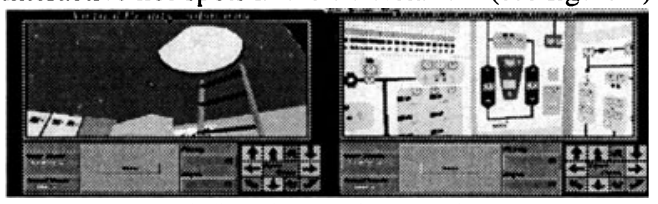

Figure 1: left: difficulties getting precise positioning to navigate through the hatch at the top of the ladder right: only some dials/buttons on the switchboard are active but there is no clear indication which they are

Similar problems have been found in other evaluation studies of VEs, such as Miller (1994). Therefore, major interaction problems exist with current VEs and these 
problems appear to be very different to the typical problems found with conventional user interfaces (e.g. Sutcliffe and Springett 1992), thus supporting hypothesis 1 .

In the second study, $10 \mathrm{VE}$ designers participated in structured interviews, to gather information about their approaches to design and issues and problems encountered in design. Designers lacked a coherent approach to design, especially interaction design. They were preoccupied with technical difficulties and thought little about supporting user interaction. There was a lack of understanding of the concepts underlying VEs and conventional HCI methods/guidelines were not used.

Therefore, the designer study indicates there is a need to support the process of interface design with guidance specific to VEs, further supporting hypothesis 1 .

\section{A THEORY OF INTERACTION}

To develop interface design guidance, an understanding of user interaction is required. Therefore, a theoretical model of interaction in VEs has been developed and is complete.

Previous work in interaction modelling has involved various approaches, such as the use of cognitive architectures (e.g. in the AMODEUS project, Barnard 1991), process models (e.g. Norman 1988) that describe interaction at a higher level of granularity, and models of user knowledge and its use (e.g. CCT, Kieras and Polson, 1985).

The approach here has been to begin with process modelling which describes interaction at a suitable level of granularity. Norman's generalised model of action was used and elaborated by adding further stages of activity to describe interactive behaviour in VEs. The elaboration of Norman's model was based on characteristics of VEs theorised to impact interaction behaviour.

Tasks in VEs are often loosely structured with exploration and opportunistic actions (Hayes-Roth and Hayes-Roth 1979). For example, in many simulation and tutorial applications, the user's task is to explore the environment so behaviour is primarily opportunistic following of cues. VEs are often active, with objects operating independently of the user's actions through system initiative (Bryson, 1995). Therefore, 3 interconnected cycles have been used to model interaction:
Task/action cycle - describes purposeful, top-down behaviour in planning and carrying out specific actions as part of the user's task or goal, and then evaluating the success of actions.

Explore/navigate cycle - describes opportunistic and less goal directed behaviour when the user explores or searches for features of interest.

System initiative cycle - describes reactive behaviour to prompts and events, and to the system taking interaction control from the user (e.g. taking the user on a pre-set tour of the environment).

Figure 2 shows a section of the explore/navigate cycle. The user scans the environment with either an intention to explore or an intention to search for an object to carry out actions from the task/action cycle. The user then continually navigates and scans until target objects are found or features in the environment arouse interest, possibly prompting exploratory actions.

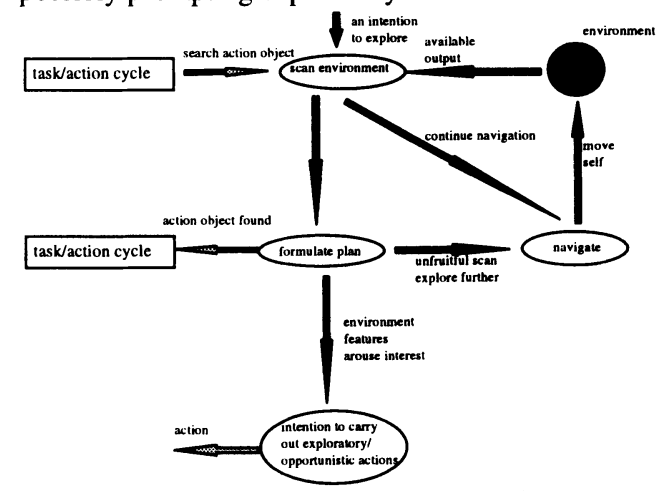

Figure 2: Section of explore/navigate cycle

Complementary to the interaction cycles, 6 knowledge sources are identified which together describe important information which is potentially available to the user during interaction. The sources are the part of the VE currently perceivable, the user's internal model of the $\mathrm{VE}$, knowledge of the domain the VE is modelled on, knowledge of the user task, knowledge about real world phenomena (e.g. function/appearance of a door), and, standards and commonalities between VEs.

To summarise, a theory of interaction has been developed predicting general interaction behaviour and relevant sources of knowledge. To be useful, the theory must be accurate and representative of actual interaction behaviour and lead to usability principles for VEs. 


\section{REQUIRED DESIGN PROPERTIES}

The interaction models provide a clear breakdown of interactive behaviour. The models have been used to systematically reason about what properties are required in a design to support the user during each identified stage in interaction, so that usability problems are avoided.

Forty-six design properties have been identified from analysis of the interaction models. The set of properties have been organised into more workable categories which are information about the user's task, spatial knowledge of the VE, the user's viewpoint and representation in the VE, objects in the vicinity, system initiative behaviour, available actions and action feedback. Table 1 lists a few of the design properties.

\begin{tabular}{|l|l|}
\hline $\begin{array}{l}\text { distinguishable } \\
\text { object/ object parts }\end{array}$ & $\begin{array}{l}\text { Objects present in the immediate vicinity can be } \\
\text { distinguished from the current environmental image. }\end{array}$ \\
\hline $\begin{array}{l}\text { identifiable object/ } \\
\text { object parts }\end{array}$ & $\begin{array}{l}\text { The object and its individual parts can be readily and } \\
\text { reliably identified. If the object is copied from real } \\
\text { world phenomena, then its representation is accurate } \\
\text { and matches user expectations. }\end{array}$ \\
\hline \begin{tabular}{l|l|} 
System initiative behaviour \\
declared system \\
control
\end{tabular} & $\begin{array}{l}\text { It is clear when the system or an agent takes control } \\
\text { of the interaction, and later when control is returned } \\
\text { to the user. }\end{array}$ \\
\hline Actions & $\begin{array}{l}\text { It is clear to the user that the potential for action } \\
\text { exists. }\end{array}$ \\
\hline $\begin{array}{l}\text { declared available } \\
\text { action }\end{array}$ & $\begin{array}{l}\text { The action can be executed efficiently and without } \\
\text { frequent obstacles/problems. The demand of } \\
\text { manipulation precision and motor co-ordination is } \\
\text { within usual human ability. }\end{array}$ \\
\hline executable action
\end{tabular}

Table 1: Example desirable design properties.

The design properties have been linked to usability problems by identifying difficulties likely to occur if the properties are missing or inadequate, or if required user knowledge is lacking. Correspondence rules have been used to specify the conditions under which usability problems are likely to occur. For example, for the exploratory action stage in the explore/navigate cycle, the design needs to make clear what actions are available with objects or the user needs to have prior knowledge of available actions. Otherwise, a predicted problem is that the user will have difficulty determining what actions are available with features in the environment they wish to investigate, as the following rule states:

IF ( (opportunities for action) NOT IN knowledge sources) AND NOT design property(declared available actions) THEN predicted problem(difficulty detecting actions available with features of interest);

\section{EVALUATING PREDICTIONS ABOUT INTERACTION - H2 \& H3}

Predictions about interaction detailed in sections 3 and 4 are being tested with ongoing experimental studies of users carrying out representative tasks with a VE developed in industry. Results will be available in July. Think aloud protocol analysis and analysis of video footage has been used to gather data on actual interaction behaviour and usability problems encountered. Observed patterns of mental and physical activity will be checked against the interaction models to test hypothesis 2 .

The data on usability problems will be used to test predictions about desirable design properties, since the importance of the properties are linked to their avoiding usability problems. The test VE has been inspected and assessed for the presence of the design properties. Predictions for usability problems have been made from inspection of the correspondence rules using the assessments of the design and expectations about likely user knowledge. Observed critical incidents and interaction breakdowns will be checked with predictions made for usability problems. A good match will support the desirable design properties in hypothesis 3 .

Results from the experiments will be used to refine the interaction models and improve predictions about required design properties.

\section{H4: THE DESIGN PROPERTIES LEAD TO IMPROVED INTERACTION}

Implementing the design properties in a VE is hypothesised to improve interaction by supporting the user during each stage of interaction and so avoiding usability problems. To test the impact of the design properties a controlled study has been planned. The study described in section 5 acts as the control condition. For the test condition, the same VE will be 'improved' by implementing missing design properties. A second set of users will then carry out the same task. Task performance is assessed for both groups with a post-study test. Improvements in interaction are defined as fewer usability problems, better task performance or lower task completion times. Hypothesis 4 will then be tested by comparing results for the 2 conditions. Results will be used to refine the set of design properties. 


\section{H5: DESIGN PROPERTIES CAN BE PRESENTED FOR VE DEVELOPMENT}

The design properties are being used to develop design guidelines and checklist questionnaires to support the design and evaluation of VEs. This is ongoing work.

Concrete design guidelines are being developed from the properties by giving for each examples, a context of use, and explanation of the guidelines effect. A hypertext tool is planned for presenting the guidelines to designers. For example, the guideline and an example for the property 'declared available action' would be:

Salient cues, prompts and affordances should be provided for available actions. Active objects and active parts of objects should be highlighted.

Example: On a switchboard, active buttons can be distinguished from inactive buttons because they protrude from the switchboard and are highlighted with an outline.

For evaluating VEs, a cognitive walkthrough method (see Polson et al. 1992) is being developed, using the interaction models and design properties. A walkthrough analysis is applied for each interaction stage by expanding the correspondence rules into question checklists for the design properties and knowledge sources required for successful interaction. For example, at the scan stage in the explore/navigate cycle the question checklist would include:

When scanning the $V E$, can the users distinguish and recognise many/few/none of the objects? Does the object appearance match the users' expectations?

Future work involves testing hypothesis 5 by using expert evaluation and critiquing of the design guidelines to gain direct qualitative feedback on the usability of the guidelines and their potential impact on the design process. The walkthrough evaluation method will be tested through direct use providing quantitative data on usability problems identified and improvements suggested. Results will be used to improve the presentation of the design properties in the design guidelines and evaluation method.

\section{CONCLUSION}

An interaction modelling approach has been proposed for addressing problems of interaction design for VEs. The models lead to design properties for supporting important stages in interaction. The benefit gained from implementation of the design properties is assessed through user studies. The theoretical research can then be applied to the problem of interaction design by using it to develop design guidelines and evaluation methods.

\section{ACKNOWLEDGMENTS}

Kulwinder Kaur is funded by the EPSRC.

\section{REFERENCES}

Barnard P. (1991). Bridging between basic theories and artefacts of Human-Computer Interaction, in Designing Interaction, Psychology at the Human Computer Interface, Ed Carroll J.M. Cambridge University Press.

Bryson S. (1995). Approaches to the successful design and implementation of VR applications, in Virtual Reality Applications, Ed Earnshaw R.A., Vince J.A. and Jones H. London, Academic Press.

Hayes-Roth B. and Hayes-Roth F. (1979). A cognitive model of planning. Cognitive Science 3, 275-310.

Kaur K., Maiden N. and Sutcliffe A. (1996). Design practice and usability problems with virtual environments, in: Virtual Reality World ' 96 conference, Stuttgart. Proceedings. IDG Conferences. Kieras D. and Polson P.G. (1985). An approach to the formal analysis of user complexity. International Journal of Man-Machine Studies, 22, 365-394.

Miller L.D. (1994). A usability evaluation of the RollsRoyce virtual reality for aero engine maintenance system. Masters Thesis. University College London. Norman D.A. (1988). The psychology of everyday things. New York, Basic Books Inc.

Polson P.G., Lewis C., Rieman J. and Wharton C. (1992). Cognitive walkthroughs: a method for theorybased evaluation of user interfaces. International Journal of Man-Machine Studies, 36, 741-773.

Rushton S. and Wann J. (1993). Problems in perception and action in virtual worlds, in Virtual Reality International 93, London. Proceedings. London, Meckler, pp. 43-55.

Sutcliffe A.G. and Springett M.V. (1992). From user's problems to design errors: linking evaluation to improving design practice, in People and Computers VII, Ed Monk A.F. and Diaper D. and Harrison M.D. Cambridge University Press. 